\title{
Introduction to the Knowledge Flows, Sharing, Transfer, and Exchange Minitrack
}

\author{
Liana Razmerita \\ Copenhagen Business School \\ $\underline{\text { ra.msc } a, c b s . d k}$
}

\author{
Lynne Cooper \\ Washington State University \\ lynnecooper.phd@gmail.com
}

\author{
Mark Nissen \\ Naval Postgraduate School \\ MNissen@,nps.edu
}

Management of knowledge flows in the Digital Age is critical to support efficient ways of working and decision support, along with innovation and entrepreneurship endeavors. The digital work environment is one in which data and information continually flow through multiple channels, within and across organizational borders (e.g. across people, groups, online communities, organizations or stakeholders). Given the uneven distribution of knowledge, harnessing the competitive power of knowledge flows requires the ability to understand and manage multiple sources of knowledge and the numerous channels used to share this knowledge. Transforming this digital knowledge flow into a sustainable competitive resource requires organizations and employees to manage dynamic knowledge processes that support sharing, interpretation (e.g., sense giving or sense making), application or knowledge creation. Knowledge platforms and systems are designed to support communication, collaboration, organizing knowledge and dynamic knowledge processes as driven by the flow of knowledge.

This minitrack examines the nature and role of knowledge flows (e.g., knowledge transfer, sharing and exchange) in the organizational context. The papers explore multiple channels (e.g, social media or social platforms, text messaging, traditional or social information systems) and widely varying domains (e.g., financial services, open source software, business intelligence, medical immunization, metal working), to shed light on the value available to organizations and individuals when people are connected efficiently to the knowledge they need.

The Call for Papers welcomed theoretical and empirical pieces addressing technical, managerial, behavioral, organizational, and economic perspectives on knowledge flows. 16 submissions were received and seven were accepted for presentation in two sessions. A brief summary of the accepted papers is presented below:

The paper "Extending a System for Measuring Dynamic Knowledge: Reconsidering Knowledge Flow Efficiency for Decision Support," by Mark Nissen, extends the state of the art in dynamic knowledge visualization and measurement to articulate a novel decision support role through the construct knowledge flow efficiency.

"An Automatic Ontology Generation Framework with An Organizational Perspective," by Samaa Elnagar, Victoria Yoon and Manoj Thomas, works to develop a novel, domain-independent, automatic ontology generation framework that converts unstructured text corpus into domain consistent ontological form.

"The Effect of Implementing an SMS Messaging System to Overcome the Lack of Transmission of HPV Facts in Saudi Arabia," by Hind Bitar, Terry 
Ryan and Sarah Alismail, utilizes an SMS messaging system to increase and maintain women's selfefficacy to educate themselves about Human papillomavirus.

"Designing Knowledge Management Systems for Supporting Craftmen's Collaboration beyond Temporal Boundaries," by Mika Yasouka and Sadayo Hirata, presents the results of a field study of knowledge transfer among craft practitioners in the metal casting industry in Japan using a prototype system developed using participatory design.

"Understanding the Impact of Knowledge Sharing through Enterprise Social Networking (ESN) on Service Innovation," by Naseem Rahman presents results from a mixed interview/survey study investigating how the use and governance of ESN for knowledge sharing in an organization affects the level of service innovation.
"Control vs Freedom: How Companies Manage their Knowledge Sharing with Open Source Software Communities," by Juliane Wisse, Michael Zaggl and Aron Lindberg, examines the micro-processes companies introduce to manage knowledge sharing with outside parties via an explorative case study conducted with Siemens AG.

"Psychology of Business Intelligence Tools: Needs-Affordances-Features Perspective," by Kaveh Abhari, Alessandro Vomero and Elizabeth Davidson, applies an Needs-Affordance-Features (NAF) framework to study the psychological motivations behind the use of different types of business intelligence tools.

The minitrack co-chairs want to thank authors and reviewers for their work in making this minitrack a success, and we look forward to continue building this research community in the coming years. 